\title{
Simulation of caspases apoptotic signalling pathway in a tuple space-based bioinformatics infrastructure
}

\author{
Maura Cárdenas-García ${ }^{\mathbb{}}$, Pedro P González-Pérez², Sara Montagna ${ }^{3}$ \\ 'Facultad de Medicina, Benemérita Universidad Autónoma de Puebla, Puebla, Mexico \\ 2Departamento de Matemáticas Aplicadas y Sistemas, Universidad Autónoma Metropolitana, México, D.F, Mexico \\ 3Dipartimento di Informatica: Scienza e Ingegneria, Univesita' degli Studi di Bologna, Cesena, Italy
}

\section{Motivation and Objectives}

Understanding intracellular communication processes is essential, since they allow the cell to perform the totality of its functions. Among them each cell has a self-destruction system that starts and operates in a regulated manner. It is called apoptosis, and includes the decision to start self-destruction as well as the proper execution of the apoptotic program. Caspases, a family of cysteine proteases, are the central regulators of apoptosis. As such, it requires the coordinated activation and execution of multiple sub programmes. Historically, different modelling approaches have been developed to deal with intracellular signalling pathways, from mathematical models - mainly Ordinary Differential Equations (ODEs) - to computational models process algebra such as stochastic $\pi$-calculus (Priami, 1995) and $\kappa$-calculus (Danos et al., 2007). Accordingly, different simulation tools have been developed, from mathematical ones - see a survey in (Alves et al., 2006) - to computational ones such as SPiM (Phillips, 2007). While they typically address scenarios with a single compartment, in recent years a trend has emerged which moves from the single global approach to mechanisms and constructs tackling the multi-compartment scenario. In this paper, we adopt a simulation approach based on the notion of Biochemical Tuple Spaces for Self-Organising Coordination (BTS-SOC), introduced in (Viroli and Casadei, 2009), and then show how it can be applied to the simulation of the caspases signalling pathway (MacFarlane and Williams, 2004), which plays a crucial role in the transduction and execution of the apoptotic signal induced by various stimuli.

\section{Methods}

A Biochemical Tuple Space for Self-Organising Coordination (BTS-SOC) is a tuple space working as a compartment where biochemical reactions take place, chemical reactants are represented as tuples, and biochemical laws are represent- ed as coordination laws by the coordination abstraction. Technically, biochemical tuple spaces are built as ReSpect (Reaction Specification Tuples) tuple centres (Omicini and Denti, 2001), running upon a TuCSON (Tuple Centres over the Network) coordination infrastructure (Omicini and Zambonelli, 1999). Tuples are logic-based tuples, while biochemical laws are implemented as ReSpect specification tuples. In particular, each biochemical tuple space is built around a ReSpect chemical engine, whose core is an action selection mechanism based on Gillespie algorithm (Gillespie, 1977) - an algorithm typically used to simulate systems of chemical/biochemical reactions efficiently and accurately - to execute chemical reactions with the proper rate. The main components of our BTS-SOC model for simulating intracellular signalling pathways are the following: 1) tuple centres - representing extracellular milieu and intracellular compartments, i.e., extracellular space, membrane, cytosol, nucleus and mitochondria; 2) chemical reaction sets - modelling signalling components, i.e., proteins (membrane receptors, enzymes, regulators, adapters, etc.) and genes; and 3) elements recorded as tuples in a tuple centre representing signalling molecules, e.g., ATP, inorganic phosphate, second messengers, etc.

The work reported here represents the initial approach to the simulation of the caspases apoptotic signalling pathway. The work was performed as follows:

1.Review of the literature involving the caspases pathway and experimental kinetic data of them in humans (Roschitzki-Voser et al., 2012; Chowdhury et al., 2008).

2.Modelling the signalling components-e.g., chemical reactions-belonging to the caspases apoptotic signalling pathway. We start with a minimalist model where each signalling component is described by the following attributes: 1) identity; 2) concentration in each cellular compartment; 3) free concentration; 4) "bound" 
concentration; 5) cellular compartment to which it belongs; 6) chemical reactions involving the component and the order in which they occur according to the affinity of the components; and 7) reaction temporality situation.

3. Simulation of the caspases apoptotic signalling pathway in the BTS-SOC-based bioinformatics infrastructure.

3.1. Creating cellular compartments. A tuple centre (BTS) is required for each cellular compartment involved in the signalling pathway to be simulated. In our study, four tuple centres (membrane, cytosol, mitochondria and nucleus) are required to model four intracellular compartments.

3.2. Introducing reactants. In order to set up the simulation system, reactants should be introduced in the BTS. First of all, each reactant belongs to a specific cellular compartment-so, it has to be put in the appropriate BTS. Initially, only the pre-existing reactants - i.e., those reactants al- ready in the compartments before the signalling pathway is activated - have to be put in the BTS.

3.3.Setting chemical reactions. The last step in setting up the simulation is the introduction of the reactions modelling the behaviour of signalling pathway, In our model, based on the Gillespie algorithm, every chemical reaction has a rate that expresses (along with the concentration of the input elements) the probability of the transformation.

4. Getting simulation outcomes. After entering all required information and setting the initial parameters, the system is now ready to run the caspases apoptotic pathway simulation.

5. Analysis and parameter adjustment.

\section{Results and Discussion}

Our modelling and simulation methodology initially considers the caspases signalling pathway as shown in Figure 1A. The pathway begins with the death signals (hormones, growth factors, cytokines, stress, etc); these signals trigger two

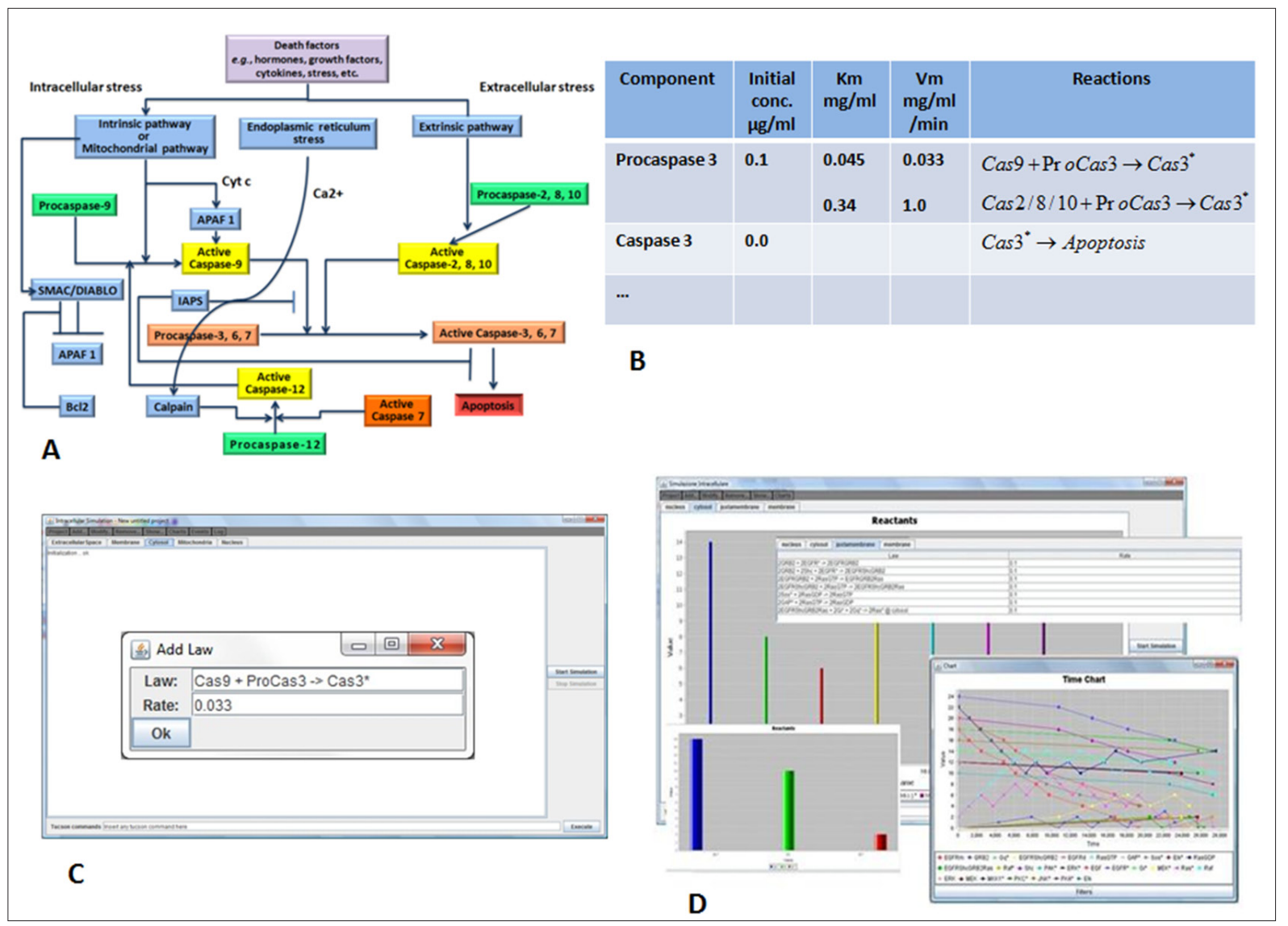

Figure 1. Basic steps of our modelling and simulation methodology. A. the effectors caspases 3, 6, 7 are activated as a consequence of the activation of the extrinsic or intrinsic pathway. B. Modelling of a signalling segment, from procaspase 3 to caspase $3^{*}$ C. Setting chemical reactions. D. Run the simulation and visualization of results. 
types of response through extrinsic and intrinsic pathways. The modelling and simulation of these events are represented in Figures $1 \mathrm{~B}$ and 1C, respectively. Take just one example for the simulation, as shown in Figure 1A. The effectors caspases 3, 6, 7 are activated as a consequence of the activation of extrinsic or intrinsic pathway. Caspase-3 is critical for apoptosis and it is activated in the cytoplasm, however, two hours after being activated it can be located at the plasma membrane in the cytoplasm and nucleus. Figure ID shows the simulation results of these events in the BTS-SOC-based bioinformatics infrastructure. When running the simulation, we observe on the molecular level how cancer cells evade caspases signalling pathways, which allow us on the one hand to design an experiment and on the other to determine an invisible proteinprotein interaction, even though it is evident in the model. According to the results obtained so far, our incremental model allows us to simulate the caspases path in a manner consistent with that reported in the literature. The next step is the refinement and adjustment of the kinetic data considering the experimental results obtained by our working group.

\section{Acknowledgements}

The authors would like to thank Andrea Boccacci for making a valuable contribution to this project.

\section{References}

1. Alves R, Antunes F, Salvador A (2006) Tools for kinetic modeling of biochemical networks. Nat Biotechnol 24(6), 667-672. doi:10.1038/nbt0606-667.

2. Chowdhury I, Tharakan B, Bhat GK (2008) Caspases An update. Comp Biochem Physiol B Biochem Mol Biol 151(1), 10-27. doi:10.1016/j.cbpb.2008.05.010

3. Danos V, Feret J, Fontana W, Harmer R, Krivine J (2007) Rule-based modelling of cellular signaling. CONCUR 2007, 17-41.

4. Gillespie, DT (1977) Exact stochastic simulation of coupled chemical reactions. J Phys Chem 81(25), 23402361. doi:10.1021/j100540a008

5. MacFarlane M, Williams AC (2004) Apoptosis and disease: a life or death decision. Conference and Workshop on Apoptosis and Disease. EMBO reports 5, 674-678.

6. Omicini A, Denti E (2001) From tuple spaces to tuple centres. Science of Computer Programming 41(3), 277-294. doi: 10.1016/\$0167-6423(01)00011-9

7. Omicini A, Zambonelli $F$ (1999) Coordination for Internet application development. Auton Agent Multi Agent Syst 2(3), 251-269. Special Issue: Coordination Mechanisms for Web Agents.

8. Phillips A (2007) The Stochastic Pi Machine (SPiM). http:// research.microsoft.com/ aphillip/spim/ (accessed 5 November 2012).

9. Priami C (1995) Stochastic pi-calculus. The Computer Journal 38(7), 578-589.

10. Roschitzki-Voser H, Schroeder $T$, Lenherr ED, Frölich F, Schweizer A et al. (2012) Human caspases in vitro: Expression, purification and kinetic characterization. Protein Expr Purif 84, 236-246. doi:10.1016/j: pep. 2012.05.009

11. Viroli M, Casadei M (2009) Biochemical tuple spaces for self-organising coordination. In: Field J, Vasconcelos VT (Eds.) Coordination Languages and Models, ser. LNCS, Lisbon, Portugal: Springer, Jun. 2009, vol. 5521, 143-162. 